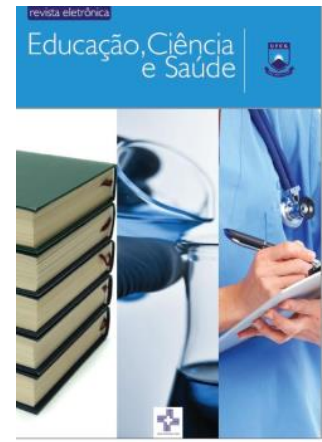

EDUCAÇÃO CIÊNCIA E SAÚDE

http://dx.doi.org/10.20438/ecs.v6i1.173

\title{
ESTUDO DO EFEITO DO PH DO BANHO ELETROLÍTICO NAS PROPRIEDADES DA LIGA NI-W OBTIDA POR ELETRODEPOSIÇÃO
}

\author{
José Anderson Machado Oliveira ${ }^{1}$, Paulo Sérgio Gomes da Silva ${ }^{2}$, Renato \\ Alexandre Costa de Santana ${ }^{2}$, Gecílio Pereira da Silva ${ }^{3}$ \\ ${ }^{1}$ Curso de Pós-Graduação em Química, Instituto de Química, Universidade Federal do Rio \\ Grande do Norte, Natal-RN, Brasil \\ ${ }^{2}$ Professor da Unidade Acadêmica de Biologia e Química, Universidade Federal de Campina \\ Grande, Cuité-PB, Brasil \\ ${ }^{3}$ Professor do Departamento de Agrotecnologia e Ciências Sociais, Universidade Federal Rural \\ do Semiárido, Mossoró-RN, Brasil \\ Email para correspondência: jmo.anderson@gmail.com
}

\begin{abstract}
Resumo
Problemas gerados pela corrosão metálica podem afetar diferentes setores industriais gerando elevados prejuízos econômicos. Revestimentos metálicos têm sido utilizados para inibir ou proteger diferentes materiais contra a corrosão, dentre esses revestimentos, destacam-se a utilização de ligas de tungstênio com metais do grupo 8B (Fe, Ni e Co). Neste trabalho estudou-se o efeito da variação do pH do banho eletrolítico para obtenção da liga de Ni-W. Composição química, morfologia superficial e ensaios eletroquímicos de corrosão foram realizados. A variação no pH do banho eletrolítico teve influência direta na composição química da liga, sendo observada uma elevação na proporção percentual em massa de tungstênio com a diminuição do $\mathrm{pH}$. A proporção de tungstênio e a morfologia do depósito influenciaram nas propriedades de resistência à corrosão da liga de Ni-W. Portanto, esperamos que a liga obtida neste trabalho possua propriedades promissoras para aplicações em diferentes setores tecnológicos e industriais.
\end{abstract}

Palavras-chave: Corrosão; Eletrodeposição; Ligas de Ni-W.

\begin{abstract}
Problems caused by metallic corrosion can affect different industrial sectors generating economic losses. Metal coatings have been widely used to inhibit or protect different materials against corrosion. Among those coatings, tungsten alloys with group $8 \mathrm{~B}(\mathrm{Fe}, \mathrm{Ni}$ and $\mathrm{Co}$ ) are widely employed for various purposes. In this paper, the effect of varying the $\mathrm{pH}$ of the electrolytic bath in order to obtain-Ni-W alloys was studied. Chemical composition, surface morphology and electrochemistry corrosion, were performed. The $\mathrm{pH}$ variation in the electrolytic bath had a direct influence on the chemical composition of the alloy, in which the percentage ratio of tungsten mass increased when decreasing the $\mathrm{pH}$. The proportion of tungsten and deposit morphology had influence on the corrosion
\end{abstract}


resistance properties of the $\mathrm{Ni}-\mathrm{W}$ alloy. Therefore, we expect that the alloy obtained in this work has promising properties for applications in different technological and industrial sectors.

Keywords: Corrosion; Electrodeposition; Ni-W Alloys.

\section{Introdução}

A corrosão metálica pode manifestar-se em diferentes meios e formas. De modo geral, o processo corrosivo torna o material inadequado para sua finalidade de aplicação, causando, dessa forma, problemas frequentes em diferentes áreas e atividades industriais gerando elevados prejuízos econômicos referentes à manutenção de estruturas danificadas ou reposição de materiais destruídos pela corrosão (LIMA-NETO et al., 2006; SANTANA et al., 2007; ALIMADADI et al., 2009). Portanto, torna-se necessária a utilização de tecnologias adequadas no controle ou inibição dos processos corrosivos, no intuito de evitar ou pelo menos diminuir custos referentes a procedimentos de manutenção. Entre essas tecnologias de prevenção, destaca-se a utilização de revestimentos metálicos e compósitos obtidos por diferentes técnicas (ALJOHANAI, HAYDEN, 2013; OLIVEIRA et al., 2015; SILVA et al., 2015).

Revestimentos metálicos podem ser aplicados em diversas finalidades, entre elas, podem ser citadas a proteção anticorrosiva, aumento ou diminuição do coeficiente de atrito, aumento da dureza e resistência ao desgaste, melhoria das propriedades magnéticas e elétricas ou simplesmente para melhorar 0 aspecto decorativo do material (TSYNTSARU et al., 2009; OLIVEIRA et al., 2017). Diferentes técnicas podem ser aplicadas na obtenção de revestimentos metálicos, como, por exemplo, deposição por imersão, fusão a plasma, deposição química, metalização, eletrodeposição, entre outras. Entre as técnicas anteriormente citadas, destaca-se a eletrodeposição, pois, permite a obtenção de revestimentos de metais puros ou na forma de ligas e compósitos com composição química e propriedades físicas controladas, sobre materiais com geometrias complexas, obtidos a temperatura ambiente e utilizando equipamentos simples, garantindo, assim, uma extensa variedade de aplicações aos revestimentos obtidos (OLIVEIRA et al., 2015; OLIVEIRA et al., 2017; COSTA et al., 2018). A técnica baseia-se na deposição de um filme sobre a superfície de um material condutor pela passagem de uma corrente 
elétrica (MAZZER, AFONSO, 2012; OLIVEIRA et al., 2017). A eletrodeposição de ligas justifica-se pelas propriedades superiores àquelas oriundas da eletrodeposição de um metal puro, como exemplo, podem ser citadas as ligas de tungstênio com metais do grupo 8B (Fe, Ni e Co) (ARUNSUNAl et al., 2012; SILVA et al., 2015).

A utilização de tungstênio em ligas metálicas justifica-se devido a suas excelentes propriedades, tais como elevada dureza, elevado ponto de fusão, elevada resistência à corrosão, baixo coeficiente de expansão térmica, propriedades catalíticas, entre outras (ARMSTRONG et al., 2012; HONG et al., 2015; COSTA et al., 2018). Assim, espera-se que o tungstênio repasse tais propriedades para suas ligas. Entretanto, a literatura relata que a eletrodeposição de tungstênio puro obtido a partir de soluções aquosas contendo íons tungstato $\left(\mathrm{WO}_{4}{ }^{2-}\right)$, não tem sido conseguida com êxito. Este impedimento pode ser explicado pela formação de uma camada de óxido sobre a superfície do cátodo durante o processo de eletrodeposição (TSYNTSARU et al., 2013). No entanto, a codeposição de tungstênio com metais do grupo 8B ( $\mathrm{Co}, \mathrm{Ni}$ e $\mathrm{Fe}$ ) na presença de um agente complexante tem sido realizada com sucesso, sendo este fenômeno conhecido como codeposição induzida (BRENNER, 2007; TSYNTSARU et al., 2009; OLIVEIRA et al., 2015). Os agentes complexantes podem influenciar na microestrutura dos revestimentos, diminuindo as tensões internas no processo de formação do filme; podem influenciar também nas propriedades magnéticas e na resistência à corrosão dos revestimentos obtidos. Os complexantes mais utilizados nos processos de eletrodeposição são citratos, tartaratos, oxalatos, glicina, manitol, entre outros (BRENNER, 2007; SRIRAMAN et al., 2007; TSYNTSARU et al., 2013).

A utilização de substâncias agressivas ao meio ambiente, ou à saúde, no processo de obtenção de revestimentos metálicos por eletrodeposição vem sendo abolidas nos setores de produção tecnológicos e industriais. A utilização de cianetos nas formulações dos eletrólitos e a utilização dos revestimentos de cádmio são exemplos de substâncias que foram substituídas dos processamentos industriais devido a sua elevada toxicidade. Outro exemplo é a utilização dos revestimentos de cromo que também tem sido contestada devido à utilização de eletrólitos contendo íons cromo hexavalente $\left(\mathrm{Cr}^{6+}\right)$ que são 
carcinogênicos (KLIMENKOV et al., 2009). Outro fator limitante da utilização dos revestimentos de cromo deve-se a sua instabilidade térmica quando exposto a elevadas temperaturas (acima de $200{ }^{\circ} \mathrm{C}$ ). Do ponto de vista técnico e industrial estas limitações incentivam novas pesquisas para 0 desenvolvimento de materiais capazes de substituir os revestimentos tradicionais de cromo, além de melhorar as propriedades dos revestimentos para aplicações em condições extremas de temperatura e ambiente corrosivo (LIMA-NETO et al., 2006; LIMA-NETO et al., 2010; INDYKA et al., 2014).

O potencial hidrogeniônico $(\mathrm{pH})$ da solução eletrolítica utilizada no processo de eletrodeposição é um dos principais parâmetros influentes nas propriedades dos revestimentos obtidos. Diante disso, neste trabalho foi avaliado o efeito do pH do banho eletrolítico utilizado para obtenção da liga de $\mathrm{Ni}-\mathrm{W}$ por eletrodeposição. Foram realizados ensaios eletroquímicos de corrosão, análise de composição química, eficiência de corrente e morfologia superficial no intuito de obter revestimentos de $\mathrm{Ni}-\mathrm{W}$ que apresentem elevado potencial para diferentes aplicações industriais. Este trabalho também apresenta uma inovação na utilização de dois agentes complexantes associados no intuito de melhorar as propriedades dos revestimentos obtidos.

\section{Metodologia}

\subsection{Eletrodeposição}

Os eletrodos de trabalho utilizados no processo de eletrodeposição foram chapas de cobre com área exposta para deposição de $8,0 \mathrm{~cm}^{2}$. Antes do processo de deposição os eletrodos passaram por dois tratamentos (mecânico e químico): primeiramente a superfície dos eletrodos foi polida mecanicamente com lixas de carbeto de silício ( $\mathrm{SiC}$ ) com granulometria decrescente de 400, 600 e 1200. Em seguida, foram imersos em solução de $\mathrm{NaOH}(10 \% \mathrm{~m} / \mathrm{v})$ por cerca de 10 segundos, seguida de lavagem com água destilada, e por fim imersos em solução de $\mathrm{H}_{2} \mathrm{SO}_{4}(1 \% \mathrm{v} / \mathrm{v}), 10$ segundos de imersão, para retirar qualquer óxido formado e ativar a superfície do eletrodo (OLIVEIRA et al., 2015; OLIVEIRA et al., 2017; COSTA et al., 2018).

As condições operacionais utilizadas neste trabalho foram as seguintes: agitação catódica do eletrodo de trabalho de $30 \mathrm{rpm}$, temperatura do banho 
eletrolítico de $70{ }^{\circ} \mathrm{C}$, densidade de corrente de $50 \mathrm{~mA} / \mathrm{cm}^{2}$ controlada com 0 auxílio de um galvanostato modelo MQPG-01 da MICRO QUÍMICA, carga aplicada de $500 \mathrm{C}$ em todos os experimentos. $\mathrm{O} \mathrm{pH}$ do banho eletrolítico foi ajustado para os três valores avaliados neste trabalho: 3, 5 e 7, utilizando uma solução de $\mathrm{H}_{2} \mathrm{SO}_{4}(50 \% \mathrm{v} / \mathrm{v})$. Esses parâmetros foram definidos a partir de ensaios preliminares (não mostrados aqui) e adotados em todos os experimentos no intuito de avaliar os depósitos obtidos sob as mesmas condições experimentais.

A Tabela 1 apresenta a composição química do banho eletrolítico, preparado utilizando reagentes de grau analítico, utilizado neste trabalho.

Tabela 1: Composição química do banho eletrolítico.

\begin{tabular}{|c|c|c|}
\hline Reagente & Concentração (mol/L) & Função \\
\hline $\mathrm{NiSO}_{4}$ & 0,0150 & Fonte de níquel \\
\hline $\mathrm{Na}_{2} \mathrm{WO}_{4}$ & 0,0606 & Fonte de tungstênio \\
\hline $\mathrm{C}_{2} \mathrm{Na}_{2} \mathrm{O}_{4}$ & 0,1263 & Complexante \\
\hline $\mathrm{C}_{4} \mathrm{H}_{4} \mathrm{Na}_{2} \mathrm{O}_{6}$ & 0,1137 & Complexante \\
\hline
\end{tabular}

A célula eletrolítica utilizada no processo de eletrodeposição foi composta por dois eletrodos: eletrodo de trabalho (substrato de cobre) e uma malha cilíndrica oca de platina como contra eletrodo. Foram utilizados $80 \mathrm{~mL}$ de solução eletrolítica e todos os experimentos foram realizados em triplicata.

\subsubsection{Caracterização dos revestimentos}

As análises de composição química dos depósitos foram feitas por Energia Dispersiva de Raios X (EDX), utilizando um espectrômetro de fluorescência de raios-X por energia dispersiva da SHIMADZU, modelo EDX720. A morfologia dos depósitos foi avaliada por de Microscopia Eletrônica de Varredura (MEV) utilizando um microscópio eletrônico da TESCAN, modelo VEGA 3SBH com Detector de EDS a seco da Oxford modelo X-ACT IE150.

A eficiência de corrente do processo de eletrodeposição (EC \%) foi calculada utilizando a Equação 1 (OLIVEIRA et al., 2015; OLIVEIRA et al., 2017). 


$$
\mathrm{EC}=\frac{w}{E w * I * \frac{t}{F}}=\frac{w * F}{I * t} \Sigma \frac{c i * n i}{M i} * 100(1)
$$

Sendo $w$ a massa medida do depósito (em gramas), t o tempo utilizado para deposição (em segundos), I a corrente total utilizada no processo (em Ampère), $E W$ o peso equivalente da liga obtida (em gramas por equivalente), ci a fração em peso do elemento no depósito, ni o número de elétrons transferidos por cada átomo de metal do revestimento, $\mathrm{Mi}$ a massa atômica do elemento (em gramas por mol) e $F$ a constante de Faraday $(96,485 \mathrm{C} / \mathrm{mol}$ ).

Os ensaios eletroquímicos de corrosão foram feitos pelas técnicas de Polarização Potenciodinâmica (PP) e Espectroscopia de Impedância Eletroquímica (EIE), utilizando um postentiostato/galvanostato da AUTOLAB, modelo PG STATE 30. As curvas de PP foram obtidas com uma varredura de $\pm 300 \mathrm{mV}$ em relação ao potencial de circuito aberto (PCA), utilizando uma velocidade de varredura de $1 \mathrm{mV} / \mathrm{s}$. Os valores da resistência de polarização $\left(R_{p}\right)$ foram calculados utilizando a equação de Stern-Geary (Equação 2), utilizando o método de extrapolação das retas de Tafel (XU et al., 2015).

$$
\mathrm{Rp}=\frac{\beta a x \beta c}{2.303 j \operatorname{corr}(\beta a+\beta c)}(2)
$$

Onde $\beta a$ e $\beta c$ representam os coeficientes anódico e catódico de Tafel, respectivamente, e $j_{\text {corr }}$ representa a densidade de corrente de corrosão.

Os diagramas de EIE foram obtidos utilizando um intervalo de frequência de $10 \mathrm{kHz}$ a $0,004 \mathrm{~Hz}$ com uma amplitude de $0,01 \mathrm{~V}, 10$ pontos por década. Foi utilizado um tempo de 1200 segundos (20 minutos) para estabilização do potencial de circuito aberto (PCA).

Para a coleta dos dados e tratamento dos resultados foi utilizado um computador com o software NOVA 1.9 para as medidas de PP e EIE. Os ensaios foram feitos em meio de $\mathrm{NaCl}(0,1 \mathrm{~mol} / \mathrm{L})$, utilizando uma célula eletroquímica convencional composta por três eletrodos: calomelano saturado (ECS) como referência, platina como contra eletrodo e uma chapa de cobre revestida com a liga de $\mathrm{Ni}-\mathrm{W}$ como eletrodo de trabalho, apresentando uma 
área exposta para análise de $0,76 \mathrm{~cm}^{2}$. Os ensaios de corrosão foram realizados a temperatura ambiente de $25 \pm 2{ }^{\circ} \mathrm{C}$.

\section{Resultados}

O banho eletrolítico teve que ser solubilizado antes do processo de eletrodeposição, pois, a preparação do banho utilizando as concentrações estabelecidas neste estudo (Tab. 1) promove à formação de compostos insolúveis na faixa de $\mathrm{pH}$ entre 8 e 9 natural da solução. Para esse processo de solubilização utilizou-se uma solução de $\mathrm{H}_{2} \mathrm{SO}_{4}(50 \% \mathrm{v} / \mathrm{v})$, dessa forma, o $\mathrm{pH}$ foi ajustado para os valores avaliados neste trabalho (3, 5 e 7).

A Tabela 2 apresenta os resultados de composição química (percentual em massa, wt.\%) e a eficiência de corrente (EC \%) do processo de eletrodeposição.

Tabela 2: Composição química e eficiência de corrente da liga de Ni-W.

\begin{tabular}{|c|c|c|c|}
\hline $\mathrm{pH}$ & $\mathrm{Ni}(\mathrm{wt} . \%)$ & $\mathrm{W}($ wt.\%) & EC (\%) \\
\hline 3 & 48,573 & 51,427 & 2,126 \\
\hline 5 & 69,256 & 30,745 & 5,202 \\
\hline 7 & 85,423 & 14,577 & 4,585 \\
\hline
\end{tabular}

A Figura 1 esquematiza os resultados referentes à composição química dos depósitos obtidos nos respectivos valores de $\mathrm{pH}$ avaliados.

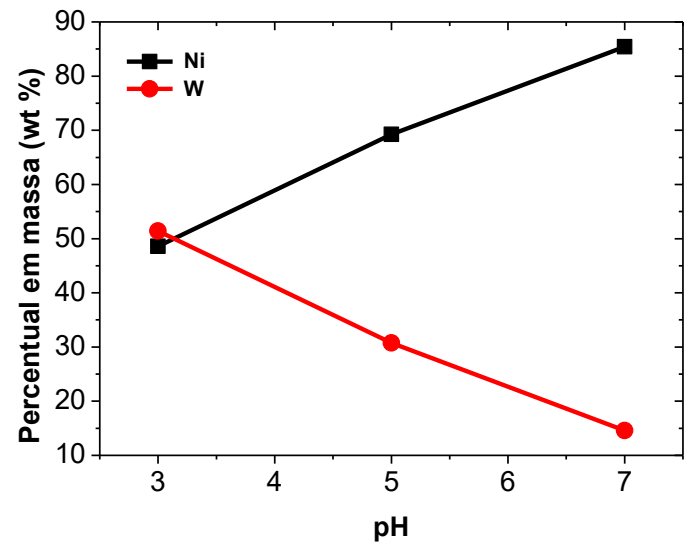

Figura 1: Composição química (wt.\%) de Ni e W nos valores de pH avaliados (Tabela 1). 
Os resultados de composição química (Fig. 1) mostram uma diminuição do conteúdo de tungstênio com a elevação do $\mathrm{pH}$ do banho eletrolítico. A diminuição do conteúdo de tungstênio nos depósitos em valores de $\mathrm{pH}$ maiores (neste caso, 5 e 7) pode ser explicado pela menor concentração de íons hidrogênio no banho nestas condições, em comparação com o banho ajustado para $\mathrm{pH}$ 3. Pois, de acordo com a literatura a reação que descreve um possível mecanismo de redução catalítica do metal tungstênio necessita da presença de íons hidrogênio para que o íon tungstato $\left(\mathrm{WO}_{4}^{2-}\right)$ reduza-se a tungstênio metálico antes de ser codepositado para formação da liga com metais do grupo 8B (Fe, Ni e Co) (TSYNTSARU et al., 2013):

$\mathrm{M}^{2+}+2 \mathrm{e}^{-} \rightarrow \mathrm{M}$

$\mathrm{WO}_{4}^{2-}+8 \mathrm{H}^{+} \rightarrow \mathrm{W}+4 \mathrm{H}_{2} \mathrm{O}$

$\mathrm{M}+\mathrm{W} \rightarrow \mathrm{M}-\mathrm{W}$

onde $\mathrm{M}$ representa um metal, indutor, do grupo 8B (Fe, Ni, Co).

Como o pH foi a variável estudada no processo de eletrodeposição da liga $\mathrm{Ni}-\mathrm{W}$, observou-se que a diminuição do mesmo favoreceu o aumento na proporção percentual em massa de tungstênio da liga obtida. Resultado semelhante foi relatado por Juškenas et al., (2009) em seu trabalho.

A Figura 2 apresenta as imagens de MEV dos revestimentos obtidos com ampliações de 1000 e 2000 vezes.
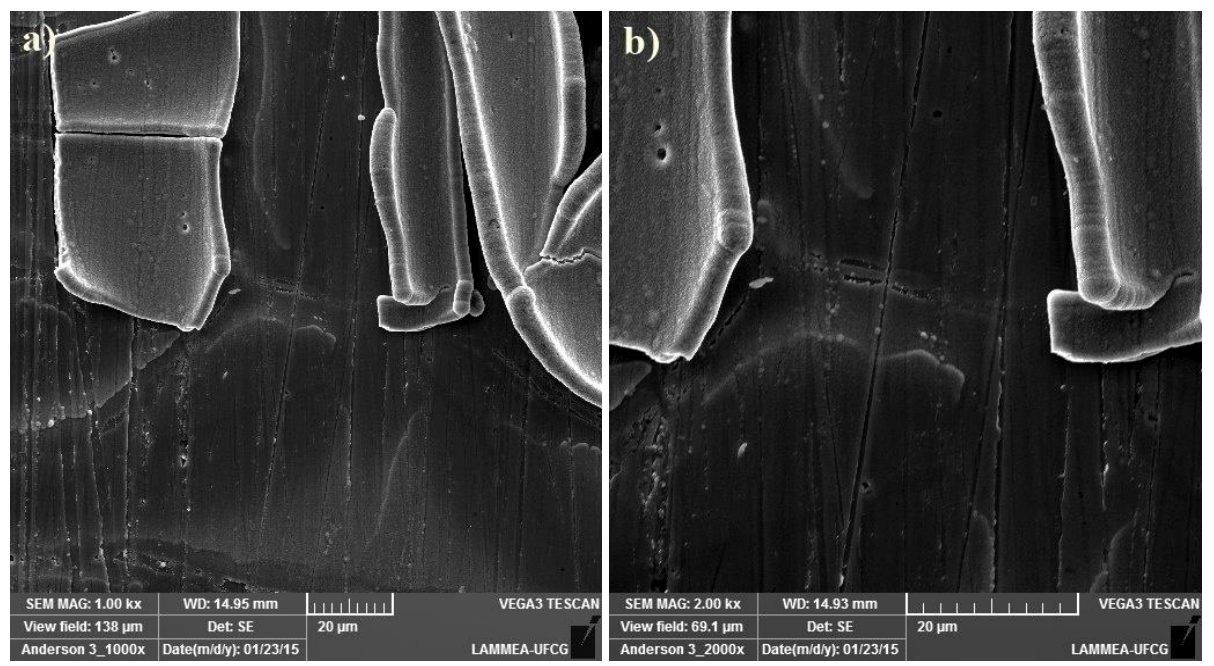

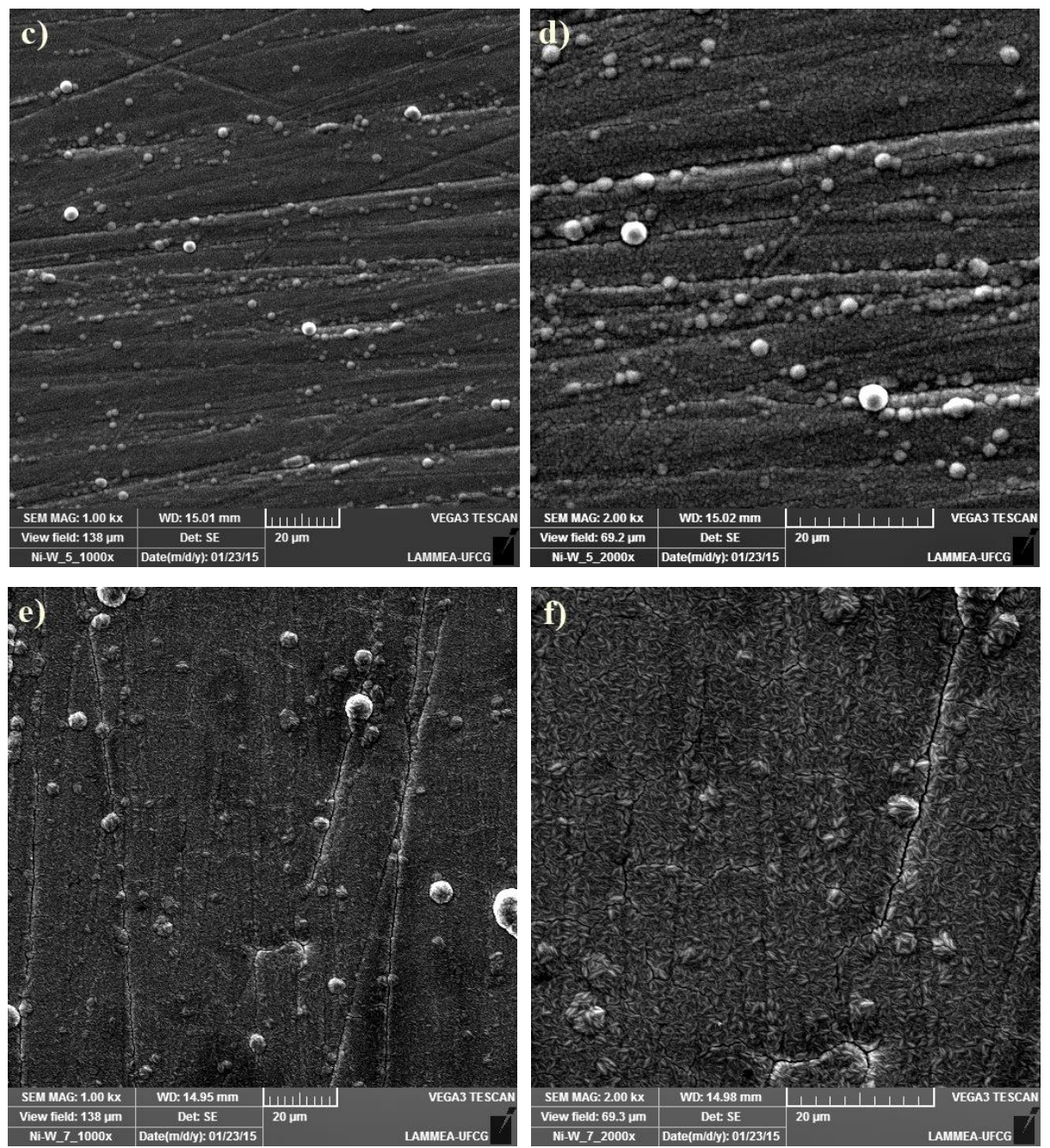

Figura 2: MEV da liga Ni-W (50 mA/cm², $\left.70{ }^{\circ} \mathrm{C}, 30 \mathrm{rpm}\right)$ : a) pH 3 (1000x); b) pH 3 (2000x); c) pH 5 (1000x); d) pH 5 (2000x); e) pH 7 (1000x) e f) pH 7 (2000x).

Observam-se diferentes morfologias nos revestimentos obtidos em diferentes valores de $\mathrm{pH}$. Nota-se a formação de camadas sobrepostas no depósito obtido em $\mathrm{pH}$ 3. Este comportamento pode estar associado ao aumento do percentual do tungstênio provocando uma elevação da tensão interna do revestimento, favorecendo, assim, a formação de trincas e o desprendimento de parte superficial do depósito. Já o depósito obtido em pH 5 apresentou uma morfologia com a formação de nódulos com geometria circular de diferentes tamanhos. Neste caso, não foi observado à presença de trincas no revestimento. $\mathrm{O}$ depósito obtido em $\mathrm{pH} 7$ também apresentou micronódulos em sua superfície, porém, a estrutura morfológica difere-se dos demais depósitos, pois apresenta uma morfologia pontiaguda semelhante aos depósitos de cromo duro (LIMA-NETO et al., 2010). 
Com o aumento do $\mathrm{pH}$ do banho eletrolítico ocorreu a diminuição do percentual de tungstênio e consequentemente um aumento no teor de níquel na liga, isso favoreceu a formação de microfissuras características de revestimentos de níquel cristalino, como evidenciado no depósito obtido em pH 7 que apresenta microfissuras em sua superfície que podem influenciar em suas propriedades de resistência à corrosão. Resultados semelhantes para morfologia de ligas de $\mathrm{Ni}-\mathrm{W}$ obtidas em diferentes condições operacionais foram relatados na literatura (SASSI et al., 2014; HAYATA et al., 2015).

A Tabela 3 apresenta os valores experimentais de potencial de corrosão ( $\left.E_{c o r r}\right)$, resistência à polarização $\left(R_{p}\right)$ e densidade de corrente de corrosão (Icorr) dos revestimentos obtidos.

Tabela 3: Parâmetros eletroquímicos de corrosão ( $E_{\text {corr }}, R_{p}$ e $\left.I_{\text {corr }}\right)$ da liga de Ni-W.

\begin{tabular}{|c|c|c|c|}
\hline pH & $E_{\text {corr }}(m V)$ & $R_{p}\left(O h m . \mathrm{cm}^{2}\right)$ & lcorr $\left(\mathrm{A} / \mathrm{cm}^{2}\right)$ \\
\hline 3 & $-284,000$ & 23836,0 & 467,430 \\
\hline 5 & $-320,830$ & 27614,0 & 312,920 \\
\hline 7 & $-402,860$ & 22920,0 & 470,760 \\
\hline
\end{tabular}

A Figura 3 mostra as curvas de Polarização Potenciodinâmica (PP) para os revestimentos de $\mathrm{Ni}-\mathrm{W}$ obtidos nos diferentes valores de $\mathrm{pH}$. Os valores do potencial de corrosão ( $\left.E_{\text {corr }}\right)$ foram calculados a partir do ponto de intersecção das curvas catódica e anódica usando o método da extrapolação das retas de Tafel. Os valores da resistência de polarização $\left(R_{p}\right)$ foram calculados utilizando a equação de Stern-Geary (Eq. 2) (XU et al., 2015). 


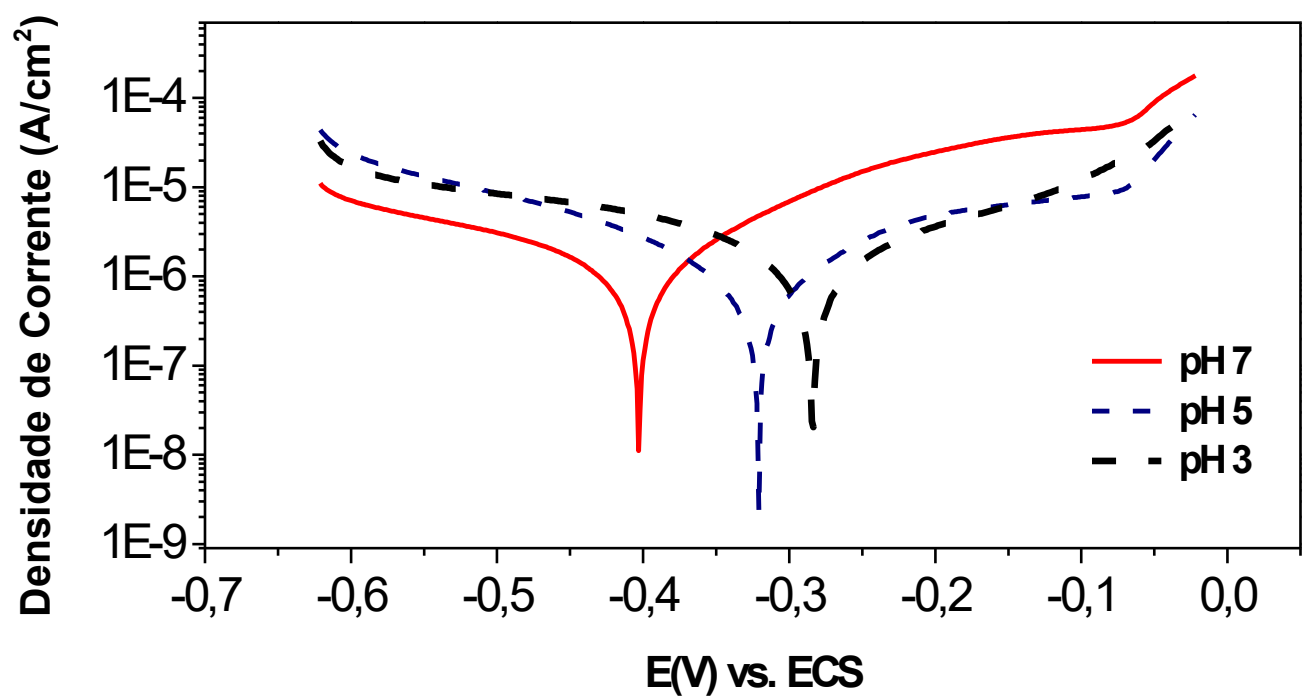

Figura 3: Curvas de Polarização Potenciodinâmica da liga de $\mathrm{Ni}-\mathrm{W}$ obtidas em $\mathrm{NaCl}(0,1$ $\mathrm{mol} / \mathrm{L}$ ) a temperatura de $25 \pm 2^{\circ} \mathrm{C}$.

De acordo com as curvas de polarização (Fig. 3), observa-se um deslocamento do potencial de corrosão para valores mais nobres à medida que a proporção de tungstênio aumenta no depósito. Isto indica que o enriquecimento deste elemento na composição química da liga acentua 0 caráter mais nobre de resistência à corrosão. Foi observado que a partir do potencial de -0,2 $\mathrm{V}$ ocorre a possível formação de um filme de passivação para os revestimentos obtidos em pH 5 e 7; tal comportamento não foi observado na liga obtida em pH 3. Observa-se uma tendência à dissolução dos revestimentos em potencial de aproximadamente -0,1 V. A menor corrente de corrosão antes do processo característico de dissolução, foi observada para a liga obtida em pH 5. Resultados semelhantes para potencial de corrosão foram relatados na literatura em estudos com ligas de Ni-W obtidas em diferentes condições operacionais (WANG et al., 2010; CHANG et al., 2011; SASSI et al., 2014).

A metodologia de polarização potenciodinâmica (PP) é um método eletroquímico eficaz para medir a taxa de corrosão. No entanto, por avaliar a taxa de corrosão por meio do acompanhamento da relação entre o potencial eletroquímico e o fluxo de corrente do processo, o valor único da taxa de corrosão pode não refletir todo o comportamento do processo corrosivo. Desta forma, com o objetivo de confirmar os resultados obtidos pelas curvas de 
polarização, foram realizados ensaios de espectroscopia de impedância eletroquímica em todos os revestimentos (SACCIA et al., 2014).

A Figura 4 mostra os diagramas de impedância de Nyquist (EIE) para as ligas de Ni-W obtidas no potencial de circuito aberto (PCA).

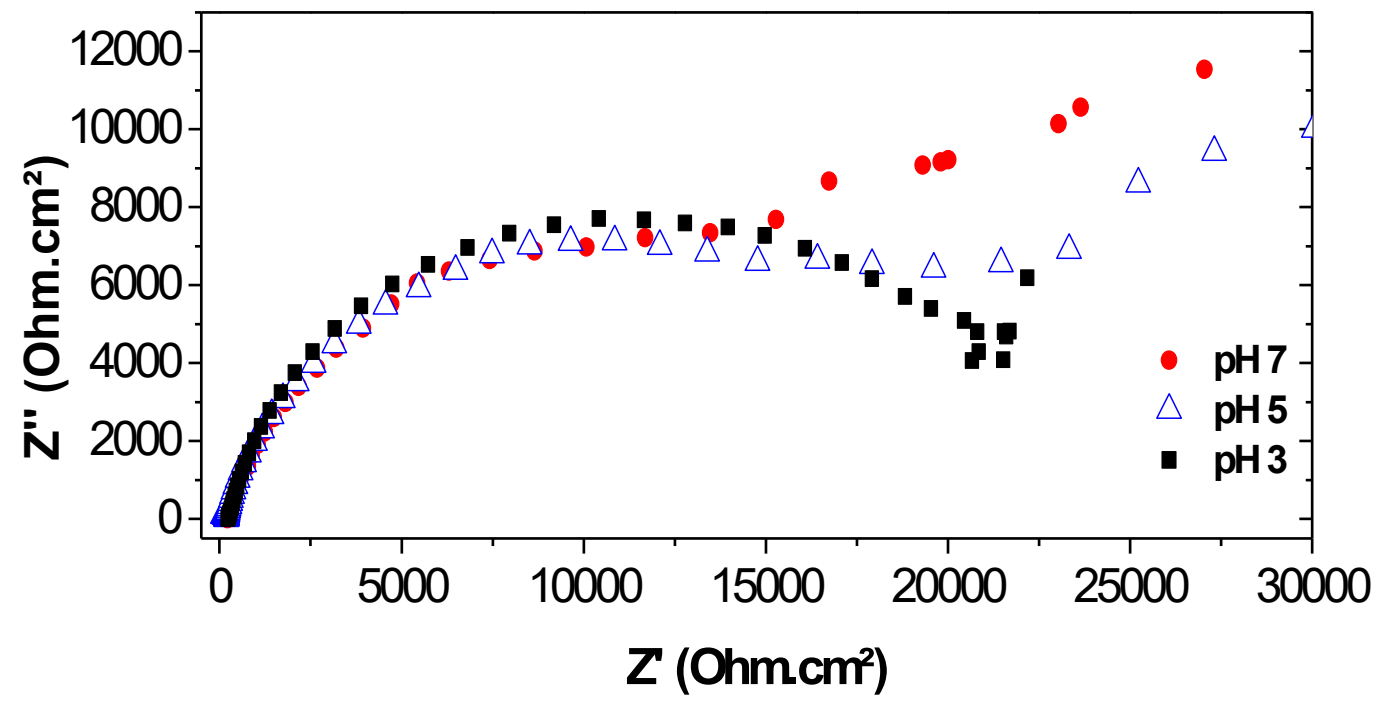

Figura 4. Diagramas de Nyquist da liga Ni-W $\left(50 \mathrm{~mA} / \mathrm{cm}^{2}, 70^{\circ} \mathrm{C}\right.$ e $\left.30 \mathrm{rpm}\right)$ : obtidas em $\mathrm{NaCl}(0,1 \mathrm{~mol} / \mathrm{L})$ a temperatura de $25 \pm 2^{\circ} \mathrm{C}$.

Observa-se para todos os revestimentos a formação de um arco capacitivo característico do processo de transferência de carga na região de alta frequência. Já na região de baixa frequência foi observado uma dispersão que pode ser associada à dissolução do revestimento em todos os experimentos. Observa-se também que todos os revestimentos apresentaram uma impedância total semelhante, evidenciado pelo tamanho aproximado de todos os diagramas. Nota-se a possível formação de um segundo arco capacitivo na região de baixas frequências nos diagramas dos revestimentos obtidos nos valores de pH 5 e 7. Segundo Chao et al., (1981), esse tipo de diagrama pode ser associado a possível formação de um filme de passivação. O comportamento observado nos diagramas de EIE está de acordo com os resultados obtidos nas curvas de $\mathrm{PP}$.

\section{Conclusão}

A liga de Ni-W pode ser obtida pela técnica de eletrodeposição com êxito seguindo as especificações citadas na metodologia deste trabalho, sendo a 
utilização dos complexantes associados necessária para obtenção de total cobertura do substrato (testes preliminares). Os depósitos recobriram toda superfície do substrato (eletrodo de cobre) e apresentaram aspecto brilhante. As micrografias apontaram diferentes morfologias dependendo do valor de $\mathrm{pH}$ utilizado para deposição: observaram-se camadas superpostas (pH 3), superfície mais homogênea com a presença de micronódulos ( $\mathrm{pH}$ ) e estrutura em forma de protuberâncias com a presença de microfissuras característica de revestimentos de níquel em estado cristalino $(\mathrm{pH} 7)$.

A proporção percentual em massa de tungstênio no depósito aumentou consideravelmente com a diminuição do $\mathrm{pH}$. A proporção do metal tungstênio na liga influenciou diretamente nos valores de resistência à polarização, densidade de corrente de corrosão e nos potenciais de corrosão. A liga obtida em pH 3 apresentou um potencial de corrosão mais nobre em relação às demais. As ligas obtidas com pH 5 e 7 evidenciam a formação de um filme de passivação que foi confirmado com as medidas de impedância. Portanto, os resultados apresentados aqui demonstram que a liga obtida neste trabalho pode ter promissoras aplicações em diferentes setores tecnológicos e industriais.

Estudos futuros serão realizados no intuito de avaliar a resistência da liga em diferentes meios e concentrações de agentes corrosivos, além de avaliar outras propriedades mecânicas no intuito de realizar uma maior caracterização do revestimento obtido.

\section{$5 \quad$ Referências}

ALIMADADI, H.; AHMADI, M.; ALIOFKHAZRAEI, M. Corrosion properties of electrodeposited nanocrystalline and amorphous patterned $\mathrm{Ni}-\mathrm{W}$ alloy. Materials and Design, v.30, n. 4, p. 1356-1361, jun., 2009.

ALJOHANI, T.A.; HAYDEN, B.E. A simultaneous screening of the corrosion resistance of Ni-W thin film alloys. Electrochimica Acta, v. 111, p. 930-936, ago., 2013.

ARMSTRONG, D.E.J.; HASEEB, A.S.M.A.; ROBERTS, S.G.; BADE, W.K. Nanoindentation and micro-mechanical fracture toughness of electrodeposited 
nanocrystalline Ni-W alloy films. Thin Solid Films, v. 520, n. 13, p. 4369-4372, fev., 2012.

ARUNSUNAI, K.K.; PARUTHIMAL, K.G.; MURALIDHARAN, V. S. Pulse electrodeposition and characterization of nano Ni-W alloy deposits. Applied Surface Science, v. 259, p. 231-237, jul., 2012.

BRENNER, A. Electrodeposition of Alloys. Applied Surface Science, v. 253, p. 7461-7466, 2007.

CHANG, L.M.; WANG, Z.T.; SHI, S.Y.; LIU, W. Study on microstructure and properties of electrodeposited Ni-W alloy coating with glycolic acid system. Journal of Alloys and Compounds, v. 509, n. 5, p. 1501-1504, nov., 2011.

CHAO, C.Y.; LIN, L.F.; MACDONALD, D.D. A Point Defect Model for Anodic Passive Films. I. Film Growth Kinetics. Journal Electrochemical Society, $v$. 128, p. 1187-1194, jan., 1981.

COSTA, J.D.; SOUSA, M.B.; ALVES, J.J.N.; EVARISTO, B.O.; QUEIROGA, R.A.; SANTOS, A.X.; MACIEL, T.M.; CAMPOS, A.R.N.; SANTANA, R.A.C.; PRASAD, S. Effect of Electrochemical Bath Composition on the Preparation of Ni-W-Fe-P Amorphous Alloy. International Journal of Electrochemical Science, v. 13, p. 2969-2985, fev., 2018.

HAYATA, S.; OUE, S.; NAKANO, H.; TAKAHASHI, T. Effect of Annealing on the Structure and Hardness of Electrodeposited Ni-W Alloys. ISIJ International, v. 55, n. 5, p. 1083-1090, jan., 2015.

HONG, S.H.; AHN, S.H.; CHOI, J. High-activity electrodeposited NiW catalysts for hydrogen evolution in alkaline water electrolysis. Applied Surface Science, v. 349, p. 629-635, ago., 2015.

INDYKA, P.; BELTOWSKA-LEHMAN, E.; TARKOWSKI, L. Structure characterization of nanocrystalline $\mathrm{Ni}-\mathrm{W}$ alloys obtained by electrodeposition. Journal of Alloys and Compounds, v. 590, p. 75-79, dez., 2014. 
JUŠKENAS, R.; VALSIUNAS, I.; PAKŠTAS, V.; GIRAITIS, R. On the state of W in electrodeposited Ni-W alloys. Electrochimica Acta, v. 54, n. 9, p. 26162620, nov., 2009.

KLIMENKOV, M.; HASEEB, A.S.M.A; BADE, K. Structural investigations on nanocrystalline Ni-W alloy films by transmission electron microscopy. Thin Solid Films, v. 517, n. 24, p. 6593-6598, abr., 2009.

LIMA-NETO, P.; CORREIA, A.N.; SANTANA, R.A.C. Morphological, structural, microhardness and electrochemical characterisations of electrodeposited $\mathrm{Cr}$ and Ni-W coatings. Electrochimica Acta, v. 55, n. 6, p. 2078-2086, nov., 2010.

LIMA-NETO, P.; SILVA, G.P.D.; CORREIA, A.N.A. A comparative study of the physicochemical and electrochemical properties of $\mathrm{Cr}$ and Ni-W-P amorphous electrocoatings. Electrochimica Acta, v. 51, n. 23, p. 4928-4933, fev., 2006.

MAZZER, E.M.; AFONSO, C.R.M. Avaliação da aplicação de recobrimento metálico amorfo para proteção contra corrosão e desgaste. Revista Eletrônica de Materiais e Processos, v. 7, p. 123-130, ago., 2012.

OLIVEIRA, A.L.M.; COSTA, J.D.; SOUSA, M.B. et al. Studies on electrodeposition and characterization of the $\mathrm{Ni}-\mathrm{W}-\mathrm{Fe}$ alloys coatings. Journal of Alloys and Compounds, v. 619, p. 697-703, set., 2015.

OLIVEIRA, J.A.M.; RAULINO, A.M.D.; RAULINO, J.L.C.; CAMPOS, A.R.N.; PRASAD, S.; SANTANA, R.A.C. Efeito da densidade de corrente e pH na obtenção da liga Ni-Fe por eletrodeposição. Revista Matéria, v. 22, n.1, set. 2017.

SACCIA, R.L.; SELAND, F.; HARRINGTONA, D.A. Dynamic electrochemical impedance spectroscopy, for electrocatalytic reactions. Electrochimica Acta, $v$. 131, p. 13-19, mar., 2014.

SANTANA, R.A.C.; CAMPOS, A.R.N.; PRASAD, S. Otimização do banho eletrolítico da liga Fe-W-B resistente à corrosão. Química Nova, v. 30, n. 2, p. 360-365, nov., 2007. 
SASSI, W.; DHOUIBI, L.; BERÇOT, P. Study of the electroplating mechanism and physicochemical proprieties of deposited Ni-W-Silicate composite alloy. Electrochimica Acta, v. 117, p. 443-452 dez.,, 2014.

SILVA, G.P.; SANTOS, A.G.; CUNHA, R.S.; SILVA, P.S.G.; OLIVEIRA, J.A.M. Avaliação de propriedades físicas e químicas de eletrodepósitos de Ni-W-P obtidos em célula de hull. Química Nova, v. 38, n. 10, 1253-1259, ago., 2015.

SRIRAMAN, K.R.; GANESH, S.R.S.; SESHADRI, S.K. Corrosion Behaviour of Electrodeposited Nanocrystalline Ni-W and Ni-Fe-W Alloys. Materials Science and Engineering, v. 460, p. 39-45, fev., 2007.

TSYNTSARU, J.; BOBANOVA, X.Y.; CESIULIS, H., et al. Iron-tungsten alloys electrodeposited under direct current from citrate-ammonia plating baths. Surface \& Coatings Technology, v. 203, p. 3136-3141, jul., 2009.

TSYNTSARU, N.; CESIULIS, H.; DONTEN, M. Modern trends in tungsten alloys electrodeposition with iron group metals. Surface Engineering and Applied Electrochemistry, v. 48, n. 6, p. 491-520, jan., 2013.

WANG, H.; LIU, R.; CHENG, F.J. Electrodepositing amorphous Ni-W alloys for MEMS. Microelectronic Engineering, v. 87, n. 10, p. 1901-1906, nov., 2010.

$X U$, J.L.; BAO, L.Z.; LIU, A.H. Effect of pore sizes on the microstructure and properties of the biomedical porous NiTi alloys prepared by microwarve sintering. Journal of Alloys and Compounds, v. 645, p. 137-142, maio, 2015.

\section{Agradecimentos}

Os autores agradecem a Coordenação de Aperfeiçoamento de Pessoal de Nível Superior (CAPES) pela bolsa concedida, ao Laboratório de Eletroquímica e Corrosão (LEC/UFCG/CES) e ao laboratório de Microscopia Eletrônica do departamento de Engenharia Mecânica (UFCG) pelas medidas de MEV. 